\title{
CAD ENVIRONMENT FOR AUTOMATED AND ROBOTIC CONSTRUCTION
}

\author{
R.J. Luke, M.M. Cusack and J.M. Jones.
}

INTERFORM (The International Forum for the Advancement of Cultural Exchanges and Technology Transfer)

Faculty of the Built Environment, University of the West of England, Bristol, BS16 1QY, United Kingdom.

\begin{abstract}
Successful robotic and automated systems require a well defined, structured and controlled work environment. It is therefore clear that before automation in the construction process can be effectively implemented, uniformity and clarity of the various tasks to be automated must be introduced. The structuring of this process must be addressed in the design phase and maintained through to completion.

Preliminary work has been undertaken at UWE on the control of a pick and place robot to construct model walls as specified by a CAD drawing. Control software is able to read an entity list of a wall design, which then calculates the number of bricks required, the orientation and position of each brick and the robot joint angles required to place each brick. This data is then used to produce an off line robot control program, enabling automatic construction of the design from a CAD drawing.
\end{abstract}

This paper discusses the next stage of this research, which is to develop further the previous work into an integrated CAD environment for buildings, which can be post processed into a robotic construction code. This would be an effective and powerful tool for guiding robots on the construction site to undertake a range of tasks associated with the construction of buildings. A CAD environment is proposed which comprises libraries of building components. These components possess individual attribute information which in addition to their physical properties can specify supplementary information, such as assembly requirements, suppliers, price and delivery. A designer can access these component libraries from within the CAD environment, select them from icon menus and insert them into the building design. This information is used as an aid when post processing the completed drawing database using assembly rules and optimisation algorithms in the production of robotic control code. It is envisaged that this database of 
information could be utilised not only to develop robotic control code, but also as a source of data to aid the integration between construction participants, by improving communication and performance. The data will also enable, for example, the automatic calculation of material requirements, estimation of times to complete work and the printing of purchase orders for materials.

\section{INTRODUCTION}

Although the construction industry is constantly evolving new practices and skills at all levels of the industry, there are clear phases within which change is either accelerated or consolidated. In short there are what Torrance [1] identifies as "cycles of growth and development which are market led and are essentially socio-economic in nature...." and also "... from time to time technological injections or political shifts [which] mark significantly important areas of development. Such are the times that we are presently entering and the pace of change is accelerating."

Given the current and projected rate of technological and organisational change, organisations engaged in construction will need to become lean, flexible, innovative and customer-driven. Most will have to re-engineer their work into streamlined and integrated business processes based on current theories and practices in relation to interorganisational relationships and the management of innovation and change. This will not only involve rethinking how to do things faster, cheaper and better but whether to do them at all.

New developments in computer based technologies such as hardware and operating systems, database management systems, computer aided software engineering, the human-computer interface, hypertext, multimedia and virtual reality will all play their part in initiating and sustaining change within the construction chain as it shifts towards becoming an integrated and information-centred manufacturing process. A recent report of the BRE concludes that the growth in information technology will lead to a substantial. culture change in the construction industry over the next five years and that all sectors of the industry face an increasing challenge to adopt an appropriate integrated IT strategy.

This particular research project involves clusters of organisations representing clients, consultants, suppliers, contractors and specialist contractors in the housing and retailing sectors of the UK construction industry. The client organisations involved are all regular users of construction's products and services and have already embarked on strategies based on partnering and the development of long term relationships with a limited number of preferred suppliers, consultants and contractors. The work of the research team is focused on developing advanced integrated computer based technologies in order to assist the participating organisations to re-engineer the construction chain associated with housing and retail projects. 


\section{REQUIREMENTS FOR CONSTRUCTION AUTOMATION}

Automated and robotic systems have proven to be an important feature in improving the effectiveness of the manufacturing industry where well defined and repetitive tasks can be specified. The experiences gained in the manufacturing industry and the decreasing cost of enabling technologies, coupled with current pressures to improve the effectiveness of the construction industry is now making the transfer of this technology into the construction chain a key element for the industry's future competitiveness. A number of construction tasks have been identified at the heart of the housing and construction sectors that have sufficient repetition to render them amenable to site based automation, these include the construction of brickwork, wall, floor and roof tiling, painting, concrete floor grinding and excavation. [2-6]. Cusack has suggested that "robotic devices must be considered an integral part of the design" [7], and that "the availability of automatic assembly will necessitate a radical revision of building design processes" [8]. This revision of the design process will need to encompass the whole of the construction chain. It will need to include consideration of the design of construction units and assembly techniques, through to the use of robotic devices to maintain and clean completed buildings.

$\mathrm{CAD}$ information is an essential source of data for integrating the construction process and for automated machines and robots in the construction industry. Drawings of building designs and site plans would provide a detailed computer understanding of what is a complex and varied workplace and of the nature of work tasks which are required to be undertaken. An array of sensory inputs and vision could provide this information, but the greater the complexity of these inputs, the greater the demand becomes on software development. Valuable processing time required to assimilate these sensory inputs would also be reduced if simple sensors can be used to confirm CAD data and provide feedback for the task at hand.

\section{METHODOLOGY}

$\mathrm{CAD}$ is widely used for the presentation of building designs, with the de facto standara being AutoCAD. For this reason it has been chosen as an appropriate medium for development of a design environment for automated and robotic construction assembly. Building components have been classified in a number of ways, including "units", "components" and "assemblies". For the purpose of this paper these terms jointly refer to any individual construction item, such as a brick, or a door frame. The building design evolves from a group of standard building components, the "block" and "attribute" features of the package have been utilised to represent the range of these components. These include window and door frames, bricks, half bricks and lintels. Each block is a scale representation of a building unit and has attribute information attached to it which specifies its physical properties and assembly rules. These blocks comprise libraries of 
building components available to the designer, which can be used to construct the building design.

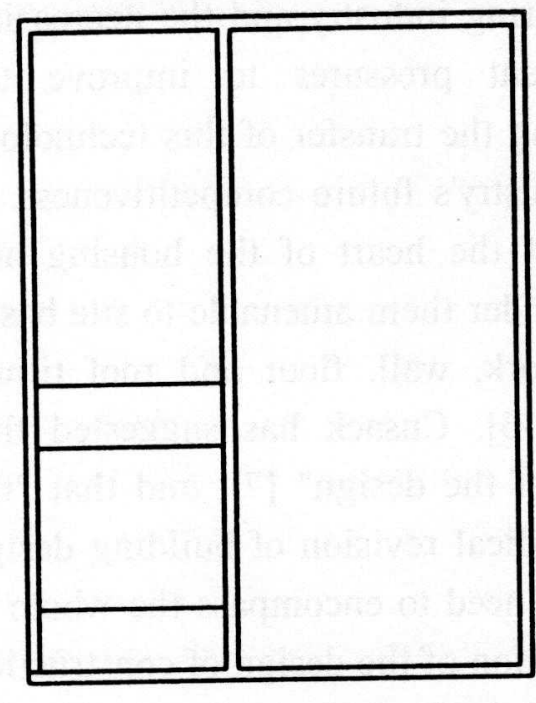

$\begin{array}{ll}\text { NAME: } & \text { Door frame DF-1A } \\ \text { PLACEMENT } & \\ \text { ORIGIN: } & \text { Lower left corner } \\ \text { X POSITON: } & ? \\ \text { Y POSITON: } & ? \\ \text { Z POSITION: } & ? \\ \text { ORIENTATION: } & ? \\ \text { MATERIAL: } & \text { Hordwood } \\ \text { HEIGHT: } & 2087 \mathrm{~mm} \\ \text { WIDTH: } & 1532 \mathrm{~mm} \\ \text { DOOR HEIGHT: } & 1981 \mathrm{~mm} \\ \text { DOOR WIOTH: } & 838 \mathrm{~mm} \\ \text { DOOR NAME: } & ?\end{array}$

Fig 1: Example of a Standard Manufacturer's Construction Unit With Appropriate Database Information.

\subsection{THE DESIGN ENVIRONMENT}

A customised design environment has been developed using the AutoCAD customization features. Both side and pull-down menus have been written which facilitate quick and easy assembly of these component parts into a final building design. Icon screens of the various components contained within the library can be toggled through and selected via mouse for subsequent insertion into the design. Editing of the final positions of these blocks is a simple matter of using the usual AutoCAD commands of copy, move, erase etc, which are all provided by the customized menus.

For the design environment to be of use to the designer, it must be possible to easily make alterations to the library of basic building units. Flexibility in utilising different components and suppliers is essential so that the product library in use suits the required standards of quality, price, delivery, etc. Repeatability in the dimensions of products is also an important requirement for automated construction.

Additional components are added to the library by constructing a drawing block which contains the required scale representation and appropriate attribute data of each building unit. A corresponding simplified drawing of the component can then be added to the icon screen with the use of a lisp program, which automatically scales and produces the required slide file. These slide files are then added to the icon slide library using AutoCAD's slidelib command. Once icons are in the library they can be utilised by the 
customized menu and pull-down screens. It is therefore a simple task to add building units to the component library and include them in the menus.

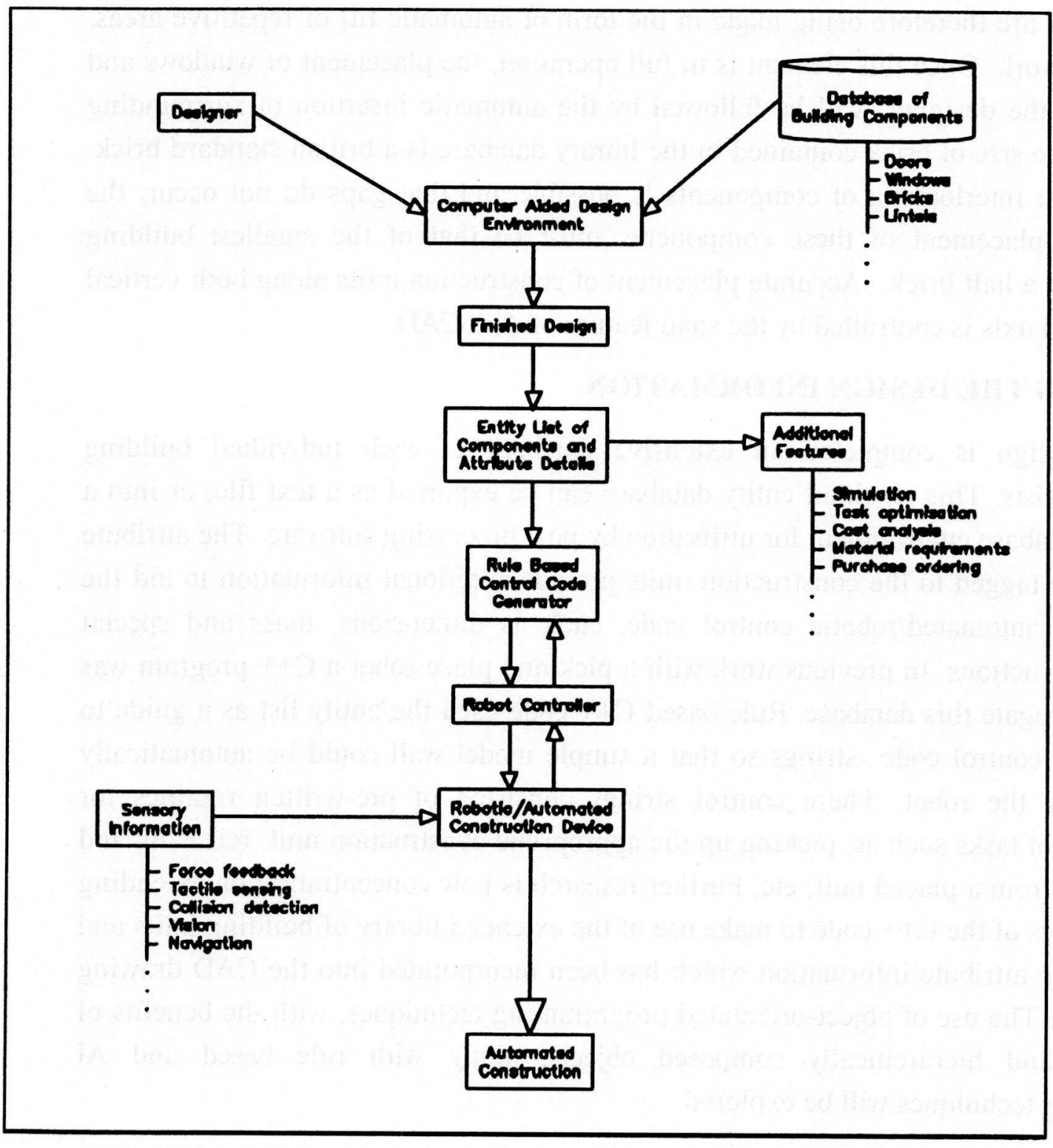

Fig:2 Structure of Automated Design and Construction Process. 


\subsection{IMPROVEMENT OF THE DESIGN ENVHRONMENT'}

The current design environment provides the means to describe a building design in terms of the orientation and position of every single component part which would be required to construct the final design. This, however, obviously makes the design stage laborious and an unsatisfactory anount of time wcilil be required to complete a design. Improvements are therefore being made in the form of autonatic fill of repetitive areas, such as brickwork. Once this element is in full opcration, the placement of windows and doors etc, by the designer, will be followed by the automatic insertion of surrounding brickwork. The size of brick contained in the library database is a british standard brick. To ensure that interlocking of components is possible and that gaps do not occur, the resolution of placement of these components must le that of the smallest building component, ie a half brick. Accurate placement of construction units along both vertical and horizontal axis is controlled by the snap feature of AutoCAD.

\section{UTILISING THE DESIGN INFORMATION}

Once the design is completed an extensive database of asil individual building component exists. This resultant cntity datalase can! be expord is a text file, or into a structured database environment for utilisation by post processing sofirare. The attribute data which is tagged to the construction !nits provide addition:al information to aid the formation of automated/robotic control code, such as diricusions, n!ass and special assembly instructions. In previous work with a pick and place robet a $\mathrm{C}++$ program was used to interrogate this database. Rule based $\mathrm{C}++$ code used the entity list as a guide to link together control code strings so that a simple model will could be automatically assembled by the robot. These control strings consisted of pre-written routines for repetitive robot tasks such as, picking up the appropriate construction unit, releasing and withdrawing from a placed unit, etc. Further research is now concentrating on extending the capabilities of the $\mathrm{C}++$ code to make use of the extended library of building units and corresponding attribute information which has been incorporated into the CAD drawing environment. The use of ebject-orientated pregramn:ing terliniques, with the benefits of inheritance and hierarchically composed objcos, alcag with rule tased and AI programming techniques will be cxplered.

\section{CONCLUSION}

There are many more factors involsed in the successful pecdurtion of automated/robotic construction than the formation of robotic control cede to assemble building units. Much active research has, and is, currently in progress in the areas of robot sensor integration, robot design, obstacle avoidance, path planning, task optimication, assembly, fixing methods, to name but a few. [9-13]. A fully autonomous construction devicc will no doubt be the result of the amalgamation of all of these. The use of a specialised CAD 
drawing environment to specify a building design can provide an invaluable wealth of data, which not only describes the building structure, but can detail individual components. The use of relational databases can extend this knowledge base for utilisation by various expert systems as listed above. It is anticipated that the CAD generated description of both the construction environment and task, will be at the centre of future robot control and decision making, as it is in CAD/CAM systems in manufacturing. The resultant $\mathrm{CAD}$ information could also be used to promote the integration of construction processes by improving communication between participants who share the common aspiration of improving performance.

\section{REFERENCES}

[1] Torrance, V.B, Forward to Male and Stocks (eds), "Competitive Advantage in Construction", Butterworth Heinemann, 1991.

[2] Warszawski A. \& Navon R, "Development of a Robot for Interior Finishing works", in Proc. 4th International Symposium on Robotics and Artificial Intelligence in Building Construction, June 22-25, 1987.

[3] Kajioka Y. \& Fujimori T, "Automating Concrete Work in Japan", in Concrete International, Pg.27-32, June 1990.

[4] Seward D. et al, "Controlling an Intelligent Excavator for Autonomous Digging in Difficult Ground", in Proc. 9th International Symposium on Automation and Robotics in Construction, Pg.743-750, June 3-5, 1992.

[5] Hisatomi Y, "Introduction of Construction Robotics in Japan", IABSE Journal, February 1990.

[6] Wing R, "Robotics in Construction: A State of the Art Review", Proc.Inst Civ.Engrs, Part 1, Pg937-952, Oct 1989.

[7] Cusack M, "Automation and Robotics: The interdependence of Design and Construction Systems", Industrial Robot Vol.21 No.4, Pg.10-14, 1994.

[8] Cusack M. \& Earl C, "Automation and Robotics: The Design and Operational Planning of Construction Projects" in Proc. 6th International Symposium on Automation and Robotics in Construction, Pg.364-370, June 6-8, 1989.

[9] Chamberlain D, Akrawi S. \& Watson J, "Path Planning and Sensing for an Experimental Masonry Building Robot", in Proc. Xth International Symposium on Automation and Robotics in Construction, Pg.229-236, June 1993.

[10] Andres et al, "First Results of the Development of the Masonry Robot System ROCCO: a Fault Tolerant Assembly Tool", in Proc. XIth International Symposium on Automation and Robotics in Construction, Pg.87-93, June 1994.

[11] Onori M, Arnstrom A. \& Ericsson A, "Practical Implementation of F.A.C.E: Flexible Assembly Control Environment", 25th ISIR, Pg.485-489.

[12] Wing R, "Assembly and Joining Facilities for Robotic Construction", in Proc. Supplement Europ IA 93, Pg.47-52.

[13] Blume C, Krisch S. \& Jakob W, "Robot Trajectory Planning with Collision Avoidance Using Genetic Algorithms and Simulation", in 25th ISIR, Pg.169-175. 